\title{
Stochastic model of 6-jet kinematic dynamo
}

\author{
Gleb Vodinchar ${ }^{1,2, \star}$ and Liubov Feshchenko ${ }^{1}$ \\ ${ }^{1}$ Institute of Cosmophysical Researches and Radio Wave Propagation FEB RAS, \\ Paratunka, Kamchatsky Kray, Russia \\ ${ }^{2}$ Vitus Bering Kamchatka State University, \\ Petropavlovsk-Kamchatsky, Russia
}

\begin{abstract}
The low-moded stochastic model of kinematic geodynamo is studied. The model is based on the indirect data about the large-scale structure of convection. The intensities of large-scale and turbulent field generators are affected by pulsed multiplicative noises. These random pulses are interpreted as the formation and destruction of coherent structures from small-scale modes of velocity and magnetic field. The perturbation of this control parameters by stochastic influence leads to switching between different dynamo regimes.
\end{abstract}

\section{Introduction}

The existing modern computing systems does not allow a direct numerical simulation of threedimensional problems of the planetary dynamo on geological time scales. The numerical models describe flows on a small-scale spatial grid at relatively small timescales $(\sim 50 \mathrm{kyr})$, or make it possible to calculate only the long-evolution large spatial structures. For the models of the second type large-scale spatial structures of convection need to be specified.

In this study, we describe a kinematic model of geodynamo with six convective cells. The study is the development of the results of work [1,2]. The following considerations are chosen for such structure of convection. Information about this structure can be obtained from the data on inhomogeneities in the density of the outer Earth's core. Results of some studies on splitting functions of the free oscillations of the Earth were analyzed in [3] and cuts of the density distribution at various depths were obtained. The splitting functions of the spheroidal mode ${ }_{11} S_{4}$ have a maximum at the liquid core depth. Corresponding density inhomogeneities are shown in Fig. 1 (left). There is a clear 12-zone structure which in the first approximation is described by a spherical harmonic $Y_{4}^{2}$.

In [3], a hypothesis on the relevant structure of convection was proposed, where the material drowns in six areas of the core and pops up in six. Then there is six convection cells (Fig. 1 (right)). It is clear that with such convection two cells are located in the equatorial region, and two cells are located still in each hemisphere. Of course, such structure of convection is very disputable. However, this hypothesis is the basis of our model.

Note that the convective structure of six cells for the liquid core of the Earth has been obtained as a result of direct numerical simulation for some value of the parameters [4]. In this work, three

\footnotetext{
^Corresponding author: gvodinchar@ikir.ru

The work was carried according to the Subject AAAA-A17-117080110043-4
} 


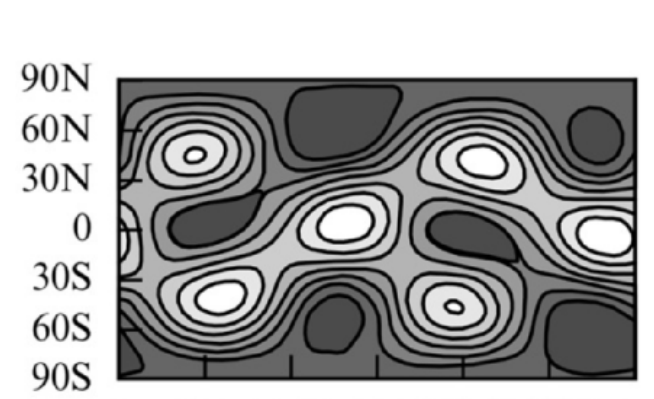

$0 \quad 60 \mathrm{E} 120 \mathrm{E} 180120 \mathrm{~W} 60 \mathrm{~W} 0$

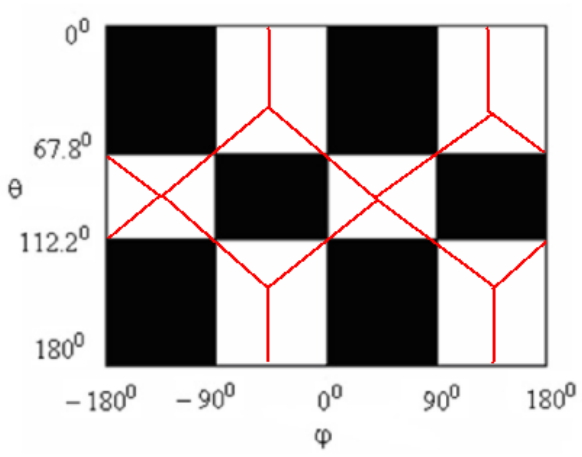

Figure 1. The map of splitting function of spheroidal mode ${ }_{11} S_{4}$ (left). The map of spherical harmonic $Y_{4}^{2}$ and the contour of convective cells (right).
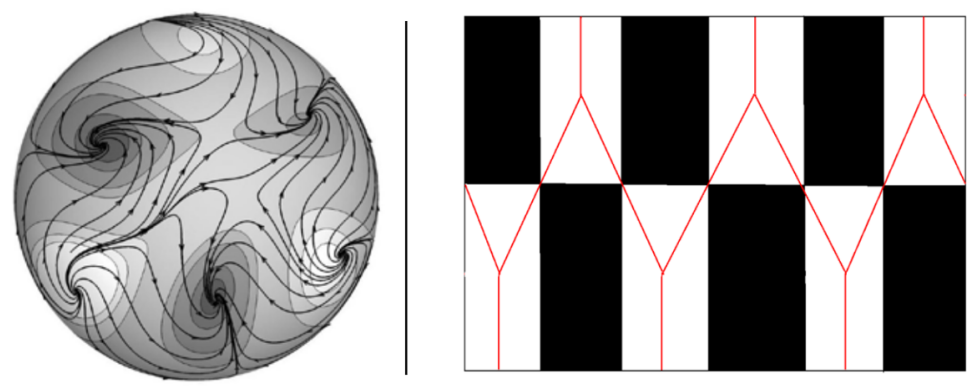

Figure 2. The tangent component of velocity on northern hemisphere [4] (left). The map of spherical harmonic $Y_{4}^{3}$ and the contour of convective cells (right).

convective cells were located in each hemisphere (Fig. 2). The poloidal component of velocity of this convection is associated with the spherical harmonic $Y_{4}^{3}$.

In this paper, we simulate the different dynamo regimes in a simple kinematic model of geodynamo. The poloidal component of velocity is determined by the spherical harmonic $Y_{4}^{2}$ (six cells). This 8-modes model with fluctuating of intensities $\alpha$-effect and large-scale generators. The source of these fluctuations can be interpreted as a result of synchronization discarded modes of velocity and magnetic field. Such spontaneous formation and destruction of the coherent structures is well known in the theory of turbulence [5].

\section{Model equations}

Consider a spherical shell of a viscous conducting liquid in a rotating coordinate system. The origin coincides with the center of the shell, and the $O z$ - axis of rotation. Velocity $\mathbf{v}$ is considered a given constant. The physical parameters of the shell is constant. We also believe that the turbulence in the shell is isotropic, and $\alpha$-effect antisymmetric with respect to the equatorial plane. Therefore we accept scalar parametrization of $\alpha$-effect in the form $\alpha(r, \theta)=\alpha_{0} a(r) \cos \theta$, where $\max |a(r)| \sim 1$ and the coefficient $\alpha_{0}>0$ determines the intensity of $\alpha$-effect. 
The dimensionless kinematic dynamo equation can be written as

$$
\begin{aligned}
& \frac{\partial \mathbf{B}}{\partial t}=\mathrm{R}_{\mathrm{m}} \operatorname{rot}\left(\mathbf{v}_{0} \times \mathbf{B}\right)+\mathrm{R}_{\alpha} \operatorname{rot}(a(r) \cos \theta \mathbf{B})+\Delta \mathbf{B}, \\
& \nabla \cdot \mathbf{B}=0,
\end{aligned}
$$

where $\mathrm{R}_{\mathrm{m}}$ - magnetic Reynolds number, and $\mathrm{R}_{\alpha^{-}}$amplitude of $\alpha$-effect. This type of dimensionless equations corresponds to the choice of outer radius $r_{o}$ of liquid core as length scale $L$, magnetic diffusion time $L^{2} / v_{m}$ as the time scale ( $v_{m}$-magnetic diffusivity) and some velocity value $U$ and the some magnetic field value $B_{0}$. For a magnetic field are set vacuum boundary conditions on the $r_{o}$.

We assume that $\mathbf{v}_{0}$ is one of the eigenmodes of the rotating liquid core free oscillations, the poloidal part of which is mainly determined by $Y_{4}^{2}$. The technique for calculating such eigenmodes is described in detail in [2].

We selected of magnetic modes by the technique proposed in [6]. Let the magnetic field is represented by a linear combination of several toroidal and poloidal modes of magnetic field free decay $\mathbf{T}_{k n}^{m}$ and $\mathbf{P}_{k n}^{m}$, where $k$ - radial index, $n$ - latitude index, and $m$ - longitude index.

The modes selection algorithm is as follows:

1. Sorting of modes at eigenvalues:

$$
\mathbf{P}_{01}^{-1.11}, \mathbf{T}_{01}^{-1.1}, \mathbf{P}_{02}^{-2.2}, \mathbf{T}_{02}^{-2 . .2}, \mathbf{P}_{03}^{-3.3}, \mathbf{P}_{11}^{-1 . .1}, \mathbf{T}_{03}^{-3 . .3}, \mathbf{P}_{04}^{-4.4}, \mathbf{T}_{11}^{-1 . .1}, \mathbf{P}_{12}^{-2.2}, \mathbf{T}_{04}^{-4.4}, \mathbf{P}_{05}^{-5.5}, \ldots
$$

2. Substitution the combination of modes $\sum_{i} g_{i}(t) \mathbf{B}_{i}(\mathbf{r})$ in the induction equation (1).

3. Apply the Galerkin method. We obtain a system

$$
\frac{d g_{k}}{d t}=\mathrm{R}_{m} \sum_{i} W_{k i} g_{i}+\mathrm{R}_{\alpha} \sum_{i} A_{k i} g_{i}-\eta_{k} g_{k}
$$

where $g_{k}(t)$ - amplitudes of the modes, and $\eta_{k}$ - eigenvalues. Matrix $W$ and $A$ are formed by Galerkin coefficients.

4. Consider the eigenvalues of matrix of the system (2). Let us call the leading and denoted as $\lambda$ it of them, which has the largest real part. Clearly, the dynamo works if and only if $\mathfrak{R e} \lambda>0$. Increasing the number of modes until the growing solutions.

5. Removing the unnecessary modes.

Note, that dynamo will be oscillating if the $\lambda$ is imaginary.

The parameters $\mathrm{R}_{m}$ and $\mathrm{R}_{\alpha}$ varied over a logarithmic scale in the ranges $\left[10^{-1} ; 10^{3}\right]$. Upon obtaining the oscillating dynamo, we discarded some modes, if this did not qualitatively change the situation and did not increase greatly the generation threshold. As a result, the magnetic modes were the following $\mathbf{P}_{0,1}^{0}, \mathbf{P}_{0,3}^{-2}, \mathbf{P}_{0,3}^{2}, \mathbf{P}_{1,1}^{0}, \mathbf{T}_{0,4}^{-2}, \mathbf{T}_{0,4}^{2}, \mathbf{P}_{0,5}^{-2}$, and $\mathbf{P}_{0,5}^{2}$.

The areas of oscillatory and non-oscillatory dynamo on the parameter plane $\left(\mathrm{R}_{m} ; \mathrm{R}_{\alpha}\right)$ for radial dependence $a(r)=\sin \frac{2 \pi\left(r-r_{i}\right)}{r_{o}-r_{i}}$ are shown in Fig. 3. This form $a(r)$ is approximate for typical radial dependence of the $\alpha$-effect computed from local simulations of weak stratified and fast rotating magnetoconvection [7]. 


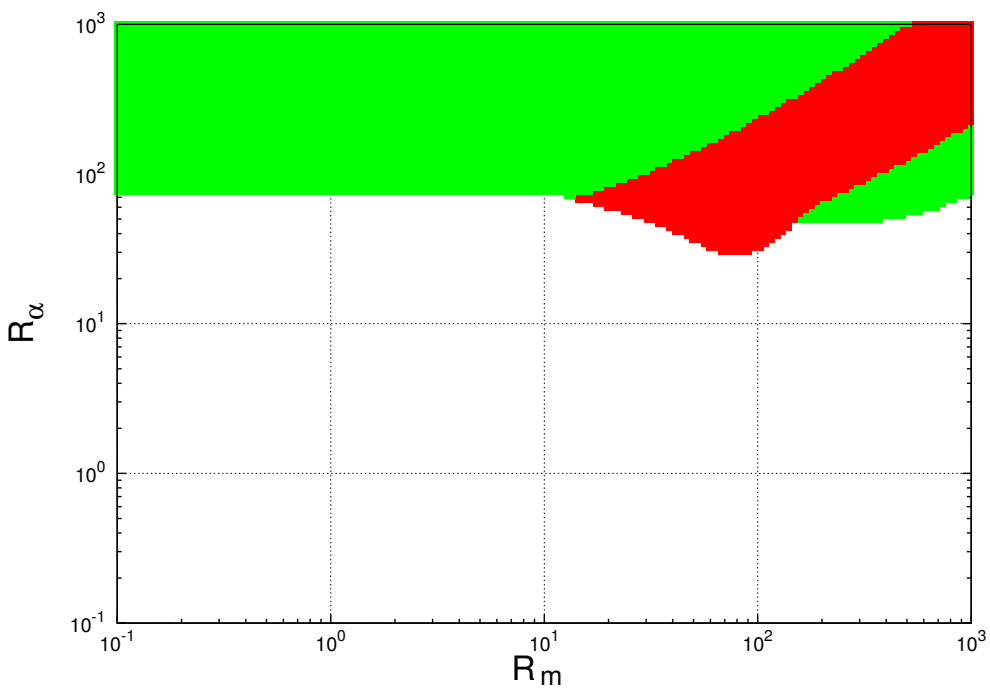

Figure 3. The areas of field generation. Red dots: non-oscillatory dynamo; green dots: oscillatory dynamo. $a(r)=\sin \left(2 \pi\left(r-r_{i}\right) /\left(r_{o}-r_{i}\right)\right)$.

Now we introduce in (2) algebraic quenching of $\alpha$-effect by field energy $\sum_{j} g_{i}^{2}(t)$. Also, introduce the multiplicative fluctuations of parameters $\operatorname{Re}_{m}$ and $\mathrm{R}_{\alpha}$ :

$$
\frac{d g_{k}}{d t}=\mathrm{R}_{m}(1+\zeta(t)) \sum_{i} W_{k i} g_{i}+\frac{\mathrm{R}_{\alpha}(1+\xi(t))}{1+\sum_{j} g_{j}^{2}} \sum_{i} A_{k i} g_{i}-\eta_{k} g_{k}
$$

where $\zeta(t)$ and $\xi(t)$ - stochastic processes whit zero means.

As mentioned above, these processes are simulate spontaneously emerging and spontaneously destroying coherence structure, formed from the discarded modes velocity and magnetic field. The structure of the processes is following.

We take of the random sequence of points $0<\tau_{1}<\theta_{1}<\tau_{2}<\theta_{2}<\cdots<\tau_{k}<\theta_{k}<\ldots$ on the time axis. We assume that the $k$-th coherent structure is formed at the time $\tau_{k}$ and destroyed at the time $\theta_{k}$. Then $T_{k}=\tau_{k}-\theta_{k-1}$ - waiting time of emergence of coherent structure, and $\Theta_{k}=\theta_{k}-\tau_{k}$ - duration of its existence. The processes $\zeta(t)$ and $\xi(t)$ are zero during the waiting time, and $\zeta(t)=\zeta_{k}$ and $\xi(t)=\xi_{k}$ during the time of existence. Here $\zeta_{k}$ and $\xi_{k}$ independent random variables with zero mean. The laws of the distribution of these variable, as well as $T_{k}$ and $\Theta_{k}$ selected in numerical simulations. The typical trajectories of processes $\xi(t)$ and $\zeta(t)$ is shown at Fig. 3.

\section{Number simulation}

Consider the some results of computational experiments with the model (3).

Note that on Fig. 3 we see two points of intersection of the separatrices. These separatrices separate the areas of the oscillating dynamo (green), the non-oscillating dynamo (red) and the area without generating (white). Then, if we take the one of these points as parameter values, then the system with the help of fluctuations may be transferred to different dynamo regimes. For one of these points $\mathrm{R}_{m} \approx 40$ and $\mathrm{R}_{\alpha} \approx 150$. It's Earth like values of parameters. 


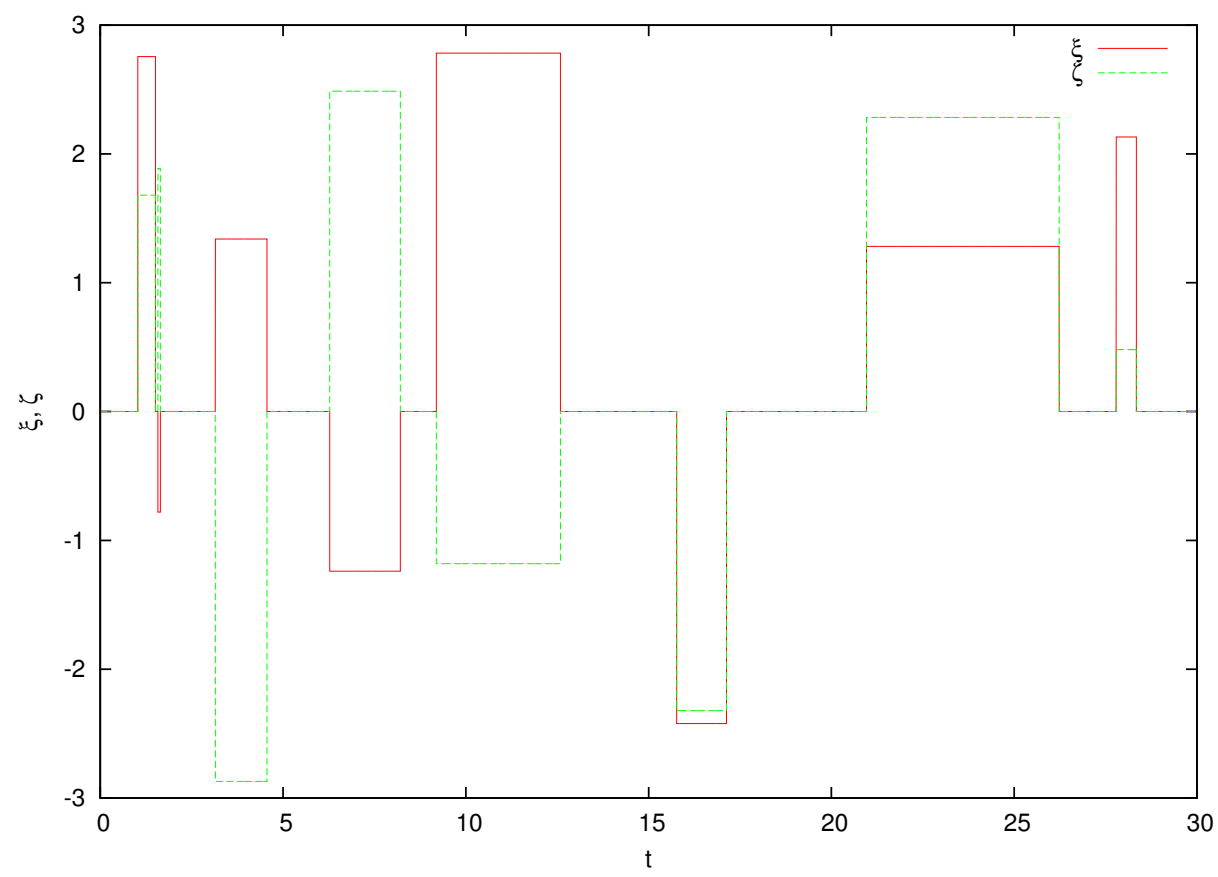

Figure 4. The typical trajectories of processes $\xi(t)$ and $\zeta(t)$.

In the simulation, we used the exponential law of distribution for the waiting time $T_{k}$ and the existence time $\Theta_{k}$, and themselves these variables were independent. Mean values $\left\langle T_{k}\right\rangle=\left\langle\Theta_{k}\right\rangle=2$.

The values of jumps $\zeta_{k}$ and $\xi_{k}$ are gaussian distributed whis the standard deviation $\sigma$.

By selecting different values of $\left(\mathrm{Re}_{m}, \mathrm{R}_{\alpha}\right)$ in a small neighborhood of a bifurcation point and different initial conditions, we have received a variety of modes dynamo: quasi-periodic, dynamobursts, the disappearance of the field, followed by growth, irregular reversals.

The results of two simulations, for example, are shown at Fig. 5 and Fig. 6.

In these figures, it is clearly seen how stable polarity regimes alternate with oscillation regimes and dynamo-bursts. At some intervals, the field almost disappears, and then the dynamo starts working again.

The source of regular reversals in the model is its internal dynamics, but violation of this cyclicality, failures in the dynamo, the yield on the regime of chaotic reversals arises due to the restructuring of the turbulence structure.

\section{Conclusion}

A low-moded stochastic model of kinematic geodynamo driven by six-cells large-scale flow in Earth's core has been developed. The model is based on the indirect data about the large-scale structure of convection.

The model contains the smallest number of modes -8 . The intensities of large-scale and turbulent field generators are affected by pulsed multiplicative noises. These random fluctuations are 

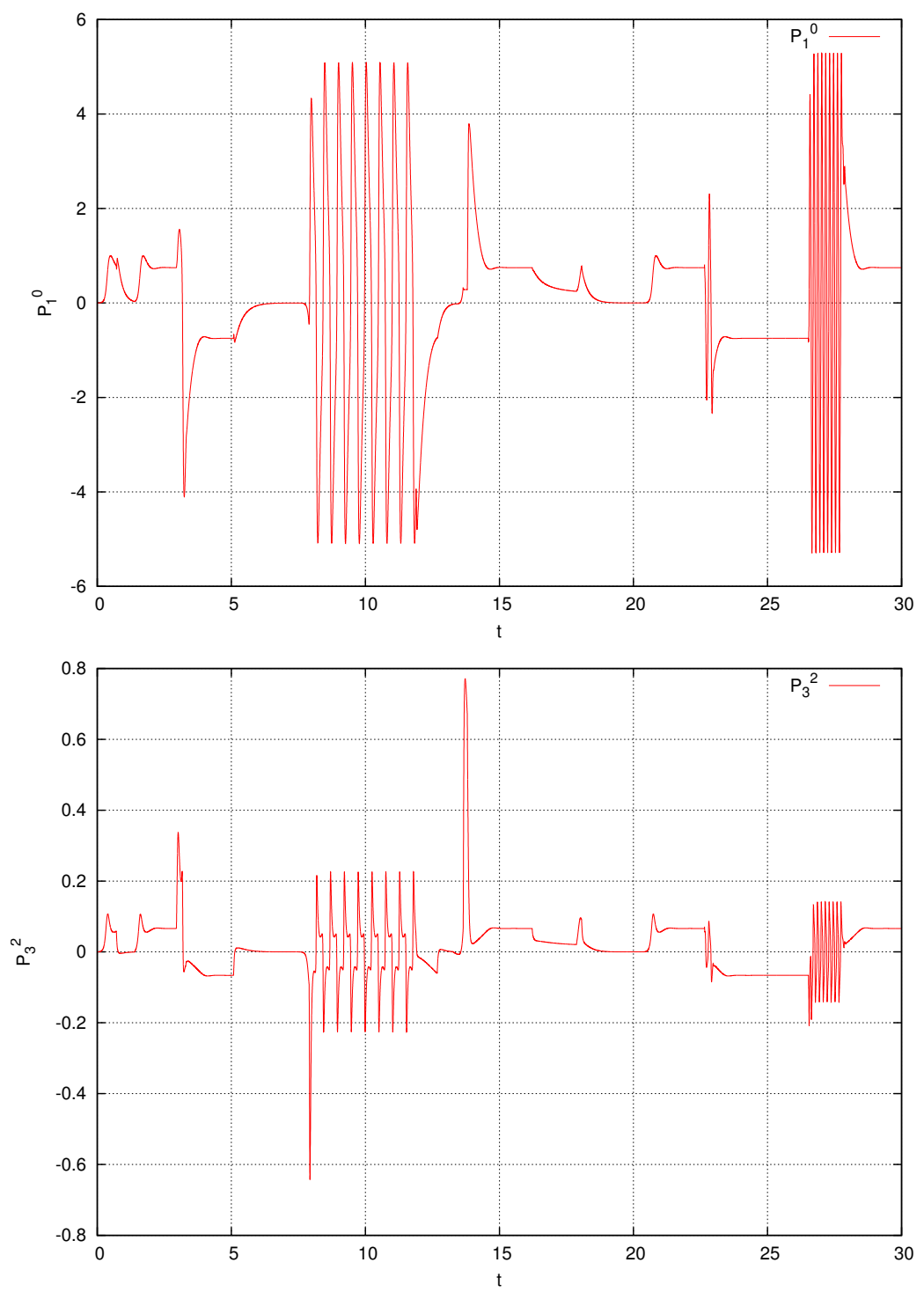

Figure 5. The amplitudes of dipole (top panel) mode, and poloidal mode $\mathbf{P}_{0,3}^{2}$ (bottom panel). Standard deviation $\sigma=3$, mean values $\left\langle T_{k}\right\rangle=\left\langle\Theta_{k}\right\rangle=2$.

interpreted as the formation and destruction of coherent structures from small-scale modes of velocity and magnetic field.

Earth like values of the magnitude of the alpha effect and the magnetic Reynolds number are locate on the boundary of the region of field generation. The perturbation of this control parameters by stochastic influence leads to switching between different dynamo regimes (stable polarity, oscillations, dynamo-bursts, quenching). 

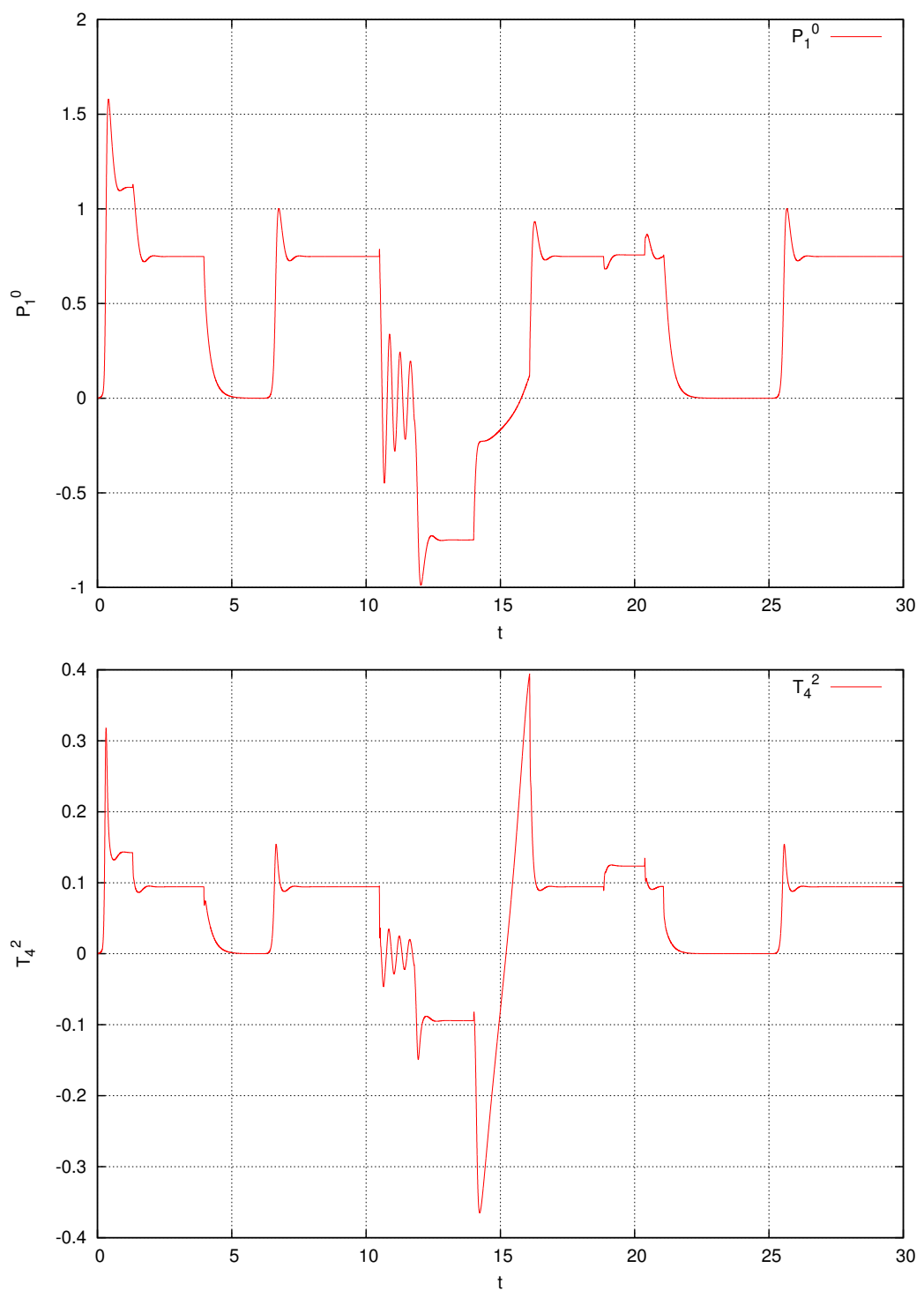

Figure 6. The amplitudes of dipole (top panel) mode, and toroidal mode $\mathbf{T}_{0,4}^{2}$ (bottom panel). Standard deviation $\sigma=1$, mean values $\left\langle T_{k}\right\rangle=\left\langle\Theta_{k}\right\rangle=2$.

\section{References}

[1] G.M. Vodinchar, L.K. Kruteva, Computational Technologies, 16(2), 35-44 (2011) (in Russian)

[2] G.M. Vodinchar, L.K. Feshchenko, Magnetohydrodynamics, 52(1), 287-299 (2016) DOI: 10.22364/mhd.52.1.32

[3] V.V. Kuznetsov, Physics-Uspekhi, 40(9), 951-961 (1997) 
[4] A.V. Gorelikov, A.V. Ryakhovsky, and A.S. Fokin. Computational Continuum Mechanics, 5(2), 184-192 (2012) (in Russian).

[5] A.V. Kolesnichenko, M.Ya. Marov, Turbulence and self-organization. Problems of modeling of cosmic and natural environments (BINOM, Moscow, 2009) (in Russian)

[6] D.D. Sokoloff, S.N. Nefedov, Numerical Methods and Programming, 8, 195-204 (2007) (in Russian)

[7] A. Giesecke, G.R. Rüdiger, D.E. Elstner, Astron. Nachr., 326(8), 693-700 (2005) DOI: 10.1002/asna.200510405 